Themenheft Nr. 45:

Pädagogisches Wissen im Lichte digitaler und datengestützter Selbstoptimierung Herausgegeben von Estella Ferraro, Sabrina Schröder und Christiane Thompson

\title{
Zur datengestützten Selbstoptimierung aus bildungstheoretischer Sicht
}

\author{
Christiane Thompson ${ }^{1}$ (D) und Estella Ferraro ${ }^{1}$ (D) \\ ${ }^{1}$ Johann Wolfgang Goethe-Universität Frankfurt
}

\section{Zusammenfassung}

Die zunehmende Implementation digitaler Technologien in pädagogische Settings wirft Fragen im Hinblick auf Selbstverhältnisse und Subjektivierung auf. Der Beitrag fragt entsprechend aus bildungstheoretischer Perspektive danach, wie digitale Technologien mit Blick auf eine datengestützte Selbstoptimierung zu einem Teil des Selbst werden. Im ersten Teil des Beitrags wird ein modernisierungstheoretischer Rahmen skizziert, der die Fragmentierung und Verflüssigung des modernen Lebens bildungstheoretisch perspektiviert. Im Anschluss daran lassen sich Prozesse der Datafizierung im Sinne einer doppelten modernen Formatierung fassen: zum einen hinsichtlich der Hoffnung auf eine doch erfüllbare Selbstbestimmung und zum anderen als ein symbolischer Riss, der sich im Medium der Daten unmittelbar wieder öffnet. Diese Struktur treibt die Datafizierung im Horizont einer sich ausweitenden «Risikoindustrie» (Rose 1999) an. Im letzten Schritt werden die Konsequenzen dieser Entwicklung für die Konstitution pädagogischen Wissens beleuchtet.

Data-Driven Self-Optimization from an Educational-Theoretical Perspective

\begin{abstract}
The ongoing implementation of digital technologies in educational settings raises questions concerning what it means to be a subject. This article is precisely concerned with the question of how digital technologies become a part of the self in and through practices of data-based self-optimization. The first part of the paper develops the fragmentation and fluidity of the self in modernity. In the second part of the paper, the authors demonstrate that datafication is related to modernity in a twofold way: It nourishes the idea of a fulfilment of the self, on the one hand, and the symbolical rupture of the self in the data, on the other hand. This structure propels datafication in the context of an increased "risk industry» (Rose 1999). The final part of the article discusses the consequences for the constitution of pedagogical knowledge.
\end{abstract}




\section{Einleitung}

Ausgangspunkt unseres Aufsatzes ist ein Kurzbeitrag des Wallstreet Journal, der 2019 erschienen ist und der sich mit der Förderung schulischen Lernens durch Künstliche Intelligenz an Schulen in China befasst. Gezeigt wird das Beispiel einer Grundschule in der Provinz Zhejiang, die Stirnbänder einsetzt, um über die Messung von Gehirnwellen Aufschluss über den Konzentrationslevel der Schülerinnen und Schüler zu erhalten. Die Lehrkraft ist mit einem Monitor ausgestattet, der es ermöglicht, das Konzentrationsniveau der gesamten Klasse, aber auch die Entwicklung der Konzentration einzelner Schülerinnen und Schüler über den Unterricht hinweg zu überwachen. Die Eltern erhalten ebenfalls Zugriff auf die Daten, so dass sie sich ein Bild von der «schulischen Arbeitsfähigkeit» ihres Kindes machen können. Von den beteiligten Eltern, Lehrkräften, Schülerinnen und Schülern ist zu erfahren, dass sich seit dem Einsatz der brain wave sensing gadgets, die Leistung der Schulklasse verbessert hat und mit grösserer Disziplin gelernt wird. Die erhobenen Daten kommen wiederum in der weiteren Forschung und technischen Entwicklung zum Einsatz.

Der Beitrag wirft eine ganze Reihe von Problemen auf, welche Datafizierung von Lernen und Bildung betreffen, darunter eine lückenlose Überwachung der Schülerinnen und Schüler, der diese nicht mehr zu entkommen vermögen, die Ausrichtung von Schule und Unterricht an der Vorstellung eines optimierten Lernens, die Öffnung des Bildungssystems für profitorientierte Unternehmen und nicht zuletzt das Problem von Datensicherheit und Datenschutz in einer Gesellschaft, die ihr Zusammenleben über ein social scoring system organisiert. Die Berichterstattung des Wallstreet Journal macht diese Probleme selbst zum Thema und lässt unter anderem einen Experten zu Wort kommen, der die Fehleranfälligkeit der Messung betont und also die wissenschaftliche Validität und Reliabilität der Daten infrage stellt. Wie nun in dem Beitrag zur Sprache kommt, entfalten die Konzentrationsmessgeräte dennoch ihre Wirkung, bestätigen doch die Lehrkräfte eine bessere Disziplin im Unterricht und einen höheren Leistungsoutput der Gruppe.

Was u. E. denkwürdig ist, lässt sich anhand von zwei Schülerkommentaren im Videobeitrag verdeutlichen. Der eine teilt den Journalistinnen und Journalisten mit, dass er im Unterricht viel aufmerksamer geworden sei und dass er nun in allen Schulaufgaben perfekte Noten erhalte (WSJ 2019, 4:15). Der andere Schüler drückt seine Besorgnis darüber aus, was passieren würde, wenn nun im Rahmen eines Tests sich herausstellte, dass man mit dem eigenen Ergebnis weit unter dem Durchschnitt liegen würde - und damit im Grunde abgehängt sei. An dieser Stelle folgt der journalistische Beitrag dem Motiv des Leistungsdrucks, das über die Technologie auf die Schülerinnen und Schüler durchschlägt. Was aber - und das ist u. E. vielsagend selbst nicht weiter zum Thema gemacht wird, ist, dass die erhobenen Daten zum Referenzpunkt der Schülerinnen und Schüler werden. Mit keinem Wort wird besprochen, was es heisst, dass die Schülerinnen und Schüler ihre Leistungsfähigkeit über 
die Technologie thematisieren. Es geht hier darum, in welcher Weise die Selbstverhältnisse im Lernen datenvermittelt sind und was sich daraus für die je eigene Vorstellung des Lernen-Könnens und der Veränderung von Selbst-Welt-Verhältnissen ergibt (vgl. dazu Breidenstein und Thompson 2014).

Mit der zuletzt genannten Formulierung begibt man sich in ein bildungstheoretisches Fahrwasser. In den Blick kommt nun nicht mehr nur das disziplinierende unterrichtliche Setting, sondern die Art und Weise, wie das etwas klobige Stirnband zu einem Teil des Selbst wird. Das Selbst konstituiert sich als eine postmediale Konstellation (Selke 2009) - mit Folgen für das, was die Schülerinnen und Schüler in den eigenen Augen werden können. In diesem Beitrag fragen wir, wie dieser Aspekt der datengestützten Selbstoptimierung aus bildungstheoretischer Sicht zu diskutieren ist. Was tragen bildungstheoretische Reflexionen bei, um die Bedeutung der Daten für Selbstverhältnisse zu reflektieren? Wir fragen dies angesichts der Einschätzung, dass die Menge der Daten, die über die Individuen gesammelt wird, zunimmt. Selke (2016a, 3, mit Bezug auf Hehl 2008) hat für diesen Umstand den Begriff der «digitalen Aura» eingebracht, welche die Individuen umgibt und ihr Innerstes nach aussen trägt. Man könnte das brain wave sensing gadget als eine Materialisierung der Idee einer solchen Aura begreifen; denn es geht mit diesem Gerät um nichts anderes als um das Erstrahlen der (innersten Substanz) des Lernens - hier: in Form einer Leuchtdiode, welche den Status der Aufmerksamkeit anzeigt - übrigens für die Schülerinnen und Schüler selbst nicht sichtbar.

Im ersten Teil unseres Beitrags skizzieren wir einen modernisierungstheoretischen Rahmen, der u. E. für das Verständnis der aktuellen Entwicklung datengestützter Selbstoptimierung unerlässlich ist. Hier geht es um die Bedeutung von Kontingenz und darum, wie daraus eine Fragmentierung und Verflüssigung des modernen Lebens hervorgeht. Dies betrifft in einer bildungstheoretisch relevanten Weise die Bestimmung des Selbst; denn es gibt kein Erkenntnisprogramm mehr, das dem Selbst erlaubt, Boden unter die Füsse zu bekommen. Das Subjekt erfährt an sich selbst Fragmentierung und Verflüssigung. Im Anschluss daran lassen sich Prozesse der Datafizierung im Sinne einer doppelten modernen Formatierung fassen: zum einen hinsichtlich der Hoffnung auf eine doch erfüllbare Selbstbestimmung und zum anderen als ein symbolischer Riss, der sich im Medium der Daten unmittelbar wieder öffnet. Diese Struktur nun, so möchten wir zeigen, treibt die Datafizierung im Horizont einer sich ausweitenden «Risikoindustrie» (Rose 1999) an. Im letzten Schritt unserer Überlegungen fragen wir nach der Bedeutung der unternommenen Analyse für pädagogisches bzw. erziehungswissenschaftliches Wissen. 


\section{Unsichere Verhältnisse: das moderne Selbst}

Dass die Moderne für das Selbst vor allem Ungewissheit bereithält, hat Zygmunt Bauman in seinen vielen Beiträgen über die «flüchtige Moderne» ausgearbeitet (Bauman 2012). Für Bauman stellt das Flüchtige in seiner fragmentierten, sich immer wieder ändernden Weise eine zentrale Metapher der Moderne dar. Flüchtigkeit und Fluidität stehen für die permanente Verschiebung von Bedeutungen und Relevanzen und für den Verlust verlässlicher Bezugspunkte in der Ausrichtung menschlichen Handelns. Was sich einerseits als Flexibilisierung der Verhältnisse verstehen lässt, führt doch andererseits dazu, dass Orientierungsmöglichkeiten verloren gehen. Das Selbst findet sich damit inmitten des Flüchtigen wieder, ist ihm doch immer wieder auferlegt, sich im Wandel der Bedeutungen und Möglichkeiten zurechtzufinden. Dieser Gedanke findet in bildungstheoretischen Reflexionen seinen Niederschlag, wenn herausgestellt wird, dass «menschliche Existenz weder nur autonom noch nur heteronom ist» (Meyer-Drawe 1990, 11). Bei aller Kritik am Souveränitätsphantasma des wissenden und sich selbst begründenden Subjekts heisst dies gerade nicht, dass die Frage der eigenen Lebensführung damit erledigt ist. Die unklare Reichweite der Selbstbestimmungsmöglichkeiten erzeugt herausfordernde Gemengelagen von Handlungs(un)fähigkeit.

Der modernisierungstheoretische und bildungstheoretische Blick entbirgt mithin, dass das moderne Selbst von Flüchtigkeit affiziert ist. Ihm bieten sich viele Möglichkeiten, einen Sachverhalt zu denken oder einen Handlungsweg einzuschlagen, ohne dass es allerdings in der Lage wäre, genau für sich zu bestimmen, was diese oder jene Möglichkeit für es bedeutet. Es ist, anders gesagt, in der Ambivalenz des «immer Andersmöglichen» gefangen, aus der heraus es sich nach Kontinuität sehnt (Bauman 2019, 3) - zwischen Vergangenheit und Zukunft. Auf dieser Grundlage bleibt die 〈Identifikation〉 des Selbst eine Fiktion. Diese Fiktion gilt gleichermassen für den Blick des Selbst auf sich wie auch für die bildungstheoretische Beschreibung des Selbst. Sich zu bestimmen, sich zu verstehen, bleibt zwar eine Aufgabe, aber es ist klar, dass diese Aufgabe nicht erfüllt werden kann. Das Selbst ist genötigt, die unvollendeten und unbefriedigenden Gefühle zwischen der Realität von dem, was ist, und der Vorstellung von dem, was sein könnte, zu verhandeln (Bauman und Vecchi 2004). In dieser Anforderung wird das Selbst bodenlos; denn es erfährt, dass die Sicherheit des Soliden ihm nicht zugänglich ist.

Die problematische Bestimmung des Selbst hat ihr Pendant in erziehungswissenschaftlichen und philosophischen Theoretisierungen des Selbst, in denen immer wieder herausgekehrt wird, dass eine einfache Identifizierung nicht möglich ist. Das Problem der Bestimmung des Selbst wiederholt sich mithin auf der wissenschaftlichen Ebene. Die Festlegung einer systematisch leitenden Kategorie zur Bestimmung des Selbst könnte den Anschein erwecken, dass das Selbst nun doch Boden unter den Füssen gewinnen könnte. Es sind seit Ende des 19. Jh. allen voran die 
existenzphilosophischen Ansätze, die zeigen, dass die menschliche Existenz nicht mehr über Wesensbestimmungen greifbar ist, dass zum Verständnis dieser Existenz vielmehr zu betrachten ist, wie die oder der Einzelne sich als Verhältnis verhält (Kierkegaard). Es gibt also eine (theoretische Unschärferelation des Selbst), die zum Beispiel Norbert Ricken (2011) dazu bewogen hat, das Selbst über die Kategorie der «Grenze» zu beschreiben. Ausgehend von der Unmöglichkeit, eine eindeutige Perspektive der Verbesserung oder Entwicklung des Selbst anzugeben, hat Alfred Schäfer (1996) die bildungstheoretisch herausfordernde Schlussfolgerung formuliert, dass sich keine Kriterien angeben lassen, die einen verlässlichen Bezugspunkt für die Überwindung entfremdeter Lebensverhältnisse oder für Mündigkeit, Selbststeigerung o. ä. bilden könnten (vgl. ebd., 277ff.). Das Fehlen der Kriterien entbindet indes nicht von der (pädagogischen) Aufgabe, Befreiung und Mündigkeit zu denken und im engen Bezug darauf einen Bildungsbegriff «nach dem Ende der humanistischen Illusion» (ebd.) auszuformulieren.

Die Wendung zum Bildungsbegriff ist nicht nur deswegen angezeigt, weil der Bildungsbegriff für das Versprechen eines selbstbestimmten Lebens steht. Es ist vielmehr nicht zu übersehen, dass die politischen und sozialen Massnahmen, die in dieser Welt der Unsicherheiten ergriffen werden, zunehmend dahin führen, die Individuen dafür verantwortlich zu machen, inmitten von Unsicherheiten und Möglichkeiten die <richtigen〉 Entscheidungen zu treffen. Sprechende Beispiele hierfür sind die beschäftigungspolitischen Massnahmen in einer flexibilisierten Arbeitswelt, die von den Individuen Eigeninitiative verlangen. Die Verantwortung für das eigene Schicksal ist also Teil des modernen Lebens (Bauman 2012) und beeinflusst die Bedingungen, unter denen das Selbst wahrgenommen wird.

Wo den Individuen deutlich wird, dass sie den sich wandelnden Verhältnissen nicht beizukommen vermögen, wird die Verantwortung für das Leben zu einer Überlastung. Es kommt zu Unsicherheiten und Ängsten, die das Individuum aushalten und - je individuell - aushandeln muss (Bauman und Vecchi 2004). Angst ist das Gefühl der Moderne überhaupt, bildet sie doch gerade die emotionale Signatur für die bereits angesprochene Bodenlosigkeit und Haltlosigkeit des Selbst (Schäfer und Thompson 2018; Thompson et al. 2021). Aus der Perspektive der Psychoanalyse lassen sich noch zwei weitere Aspekte anführen, die für unseren Blick auf die datengestützte Selbstoptimierung wichtig sind. Das ist zum einen der plötzliche Umschlag von Sicherheit in Unsicherheit - der Moment, wo es unheimlich wird (Freud).

In seinen Ausführungen zum «Selbst als Phantom» hat Michael Wimmer (2011) herausgearbeitet, wie das Selbst in seinen Positivierungsversuchen immer wieder auf Selbstfremdheit stösst. Es taucht etwas im Selbst auf, was die Vorstellung der Selbst-Kohärenz und Identität stört. Wimmer spricht mit Derrida davon, dass es im Selbst spukt (ebd., 306). Der «Phantom-Effekt» besteht nach Wimmer darin, «dass die Differenz zwischen dem (symbolischen) Geist, der das «ich〉 beseelt, und dem 
(imaginären) Ghost/Gespenst, der im Mich der Selbstrepräsentanz spukt, eingezogen wird» (ebd., 306f.). Das Unheimliche des Selbst kann nicht ausgegrenzt werden, was Erfahrungen von Verunsicherung oder sogar von Selbstverlust mit sich bringt.

Dies führt zu einer Abwehr von Angst und Selbstfremdheit. Nach Bauman und Obirek (2015) bilden die eben genannten Erfahrungen den Ausgangspunkt dafür, dass Menschen die Verantwortung für die eigenen Entscheidungen und für das eigene Handeln an jene abtreten, die ihnen politische oder wissenschaftliche Versprechungen liefern. Ob es sich bei dieser Verschiebung von Handlungsfähigkeit um eine Projektion auf Politikerinnen und Politiker handelt, von denen man glaubt, dass diese mit allen Widrigkeiten der modernen Welt fertig werden können, oder um eine Sicherheitsarchitektur, von der man sich erhofft, die Unsicherheit technologisch in den Griff zu bekommen, spielt erst einmal keine Rolle.

Aus der modernisierungstheoretischen Warte ist festzuhalten, dass der Versuch des Individuums einer Verantwortungsverschiebung eine verführende Illusion darstellt. Damit scheint ein letzter wichtiger Aspekt dieses Theoriezugriffs auf die Transformationen der Moderne auf. Es ist dies die Auffassung, dass die Verhandlungen der Ambivalenz eine politische Frage sind und bleiben. Die Ungewissheit der Moderne kann nicht herangezogen werden, um für Fatalismus oder Relativismus zu argumentieren. Bei aller Ambivalenz kommt sie doch immer wieder auf die Frage des Handelns und der Selbstpositionierung zurück. Auf dieser Grundlage schärft sie den Blick für die sozialen und politischen Realitäten der Gegenwart. Im Folgenden wollen wir dies im Umkreis von Datafizierung genauer fokussieren.

\section{Datengestützte Selbstoptimierung zwischen Schliessung und Heimsuchung}

Im Folgenden lesen wir die Prozesse datengestützter Selbstoptimierung als eine Selbsttechnologie, die von einer doppelten Bewegung geprägt ist: Sie nährt zum einen das Phantasma, dass das Phantomhafte des Selbst doch überwunden werden könnte und Selbstoptimierung im Sinne der Identität eines optimierten Selbst erreichbar ist. Zum anderen werden wir zeigen, wie die datengebundenen Repräsentationen und der Umgang mit diesen immer wieder auf den Entzug des Selbst stossen lässt, eine Heimsuchung, die ihrerseits den Drang zur Datafizierung befördert und generalisiert. Wie sich im Rahmen der Analyse auch zeigen wird, spielen in diesem Drang Naturalisierung, Ökonomisierung und Überwachung eine gewichtige Rolle.

Das Feld einer datengestützten Selbstoptimierung ist mittlerweile kaum mehr zu durchmessen. Zunächst einmal ist festzuhalten, dass die quantifizierende Erfassung menschlicher Erscheinungen, Leistungsmerkmale und Verhaltensweisen eine Geschichte hat, die, mit Foucault gesprochen, auf die biopolitische Regierung der Individuen ausgerichtet ist (Foucault 1977). Das Dispositiv der Vermessung, das im 19. Jh. Gestalt annimmt und in Form einer auf Wachstum ausgerichteten Wirtschaftsstatistik 
im 20. Jh. für das Bildungssystem relevant wird, ist sicherlich eine historisch bedeutsame Prämisse, um das Aufkommen einer Quantifizierung des Sozialen überhaupt zu verstehen (Mau 2018).

Blickt man auf die Bereiche und Bezüge, auf die sich die Quantifizierung des Selbst bezieht, wird unmittelbar deutlich, dass es kaum eine Dimension des menschlichen Lebens gibt, die von dieser Entwicklung ausgeschlossen ist. Die mittlerweile in unterschiedlichsten Fabrikaten erhältlichen Fitness-Tracker messen nicht mehr nur die Performance in der Sporteinheit. Sie überwachen ebenso den Schlaf derjenigen, die sie tragen. Sie schlagen auf der Grundlage der erfassten Daten Trainingseinheiten vor und senden Signale, wenn man zu lang unbewegt am Schreibtisch gesessen hat. Entscheidend in der Selbstoptimierung ist der relationale Vergleich mit sich selbst (z. B. der Schlaf an vorausgehenden Tagen), aus dem prospektiv Handlungsmöglichkeiten bzw. -empfehlungen abgeleitet werden. Moorstedt (2013) deutet mit seinem Text «Erscanne dich selbst!» eine entscheidende Weichenstellung an: Der Titel spielt auf die antike Formel des «Erkenne dich selbst!» vom Delphischen Orakel an. Menschen erwarten mittlerweile die für sie relevante und verbindliche Erkenntnis von und durch Daten.

Selke (2015) hat darauf hingewiesen, dass die Vorstellung einer wahren Erkenntnis des Selbst - im Gegensatz zur zuvor erwähnten Unsicherheit im Theoretisieren des Selbst - wesentlicher Ausgangspunkt für die Quantified-Self-Bewegung ist. Verfechterinnen und Verfechter dieser Bewegung würden die Quantifizierung als Chance sehen, subjektive Verzerrungen und blinde Flecken in der menschlichen Selbstwahrnehmung zu überwinden (ebd., 96). Als anschauliches Beispiel kann eine DiätApp dienen, die als verlässlicher angesehen wird als das eigene Körperbild bzw. das imaginär durchsetzte Verhältnis zum Körper. Was hier zum Ausdruck kommt, ist die Hoffnung der Rationalisierbarkeit des menschlichen Handelns - ein klassisch modernes Motiv. Daten erscheinen als ‘objektiv> und genau auf den Prozess möglicher Optimierung abgestimmt. Es ist $u$. E. eben dieser Zusammenhang, der die Vorstellung der (phantasmatischen) Erfüllung und Vollendung des Selbst nährt. Es handelt sich um das Versprechen, zur erwünschten Sicherheit im Angesicht der Unsicherheit zu gelangen.

Die Objektivierung steht in Verbindung damit, dass Daten wortwörtlich als Gegebenheit betrachtet werden. Ihnen wird der Modus des Faktischen ${ }^{1}$ zugewiesen. Darüber hinaus werden Daten in den Zusammenhang einer Kumulierung, Entwicklung, Verarbeitung und Auswertung gestellt. Dieser Zusammenhang des Datenverarbeitungsprozesses, ein entscheidender Vorverweis auf Big Data, verstärkt in kaum zu

1 Immer wieder wird in den einschlägigen fachwissenschaftlichen Diskursen darauf hingewiesen, dass Daten eben nicht einfach gegeben sind (vgl. u. a. Kitchin 2014). Dies ändert jedoch nichts daran, dass der Status von Daten zumeist auf Grundlage der Unterscheidung von «zutreffend» und «nicht zutreffend» bzw. im Modus der Wahrscheinlichkeit verhandelt wird. Die grundsätzliche Konstruktivität des Datums wird dabei aussen vorgelassen. 
überschätzender Weise den Eindruck der Objektivität: Es ist die permanente Objektivierung, welche die Bestimmung des Selbst in den rekursiven Prozess der Datenerhebung und -auswertung einkoppelt, was ein wesentlicher Motor dafür ist, Daten für das Selbst verbindlich zu machen. Mit der Zahl der Daten und der Möglichkeit ihrer Verarbeitung steigt, mit anderen Worten, ihre «Erklärungskraft», wie Behrens und DiCerbo einmal festgehalten haben, die im Bereich des educational data mining den Übergang von der «digitalen Wüste» zum «digitalen Ozean» herbeisehnen (Behrens und DiCerbo 2014).

Die Objektivierung nun scheint genau jene Ungewissheiten und Kontingenzen bezähmbar zu machen, die wir im vorausgehenden Abschnitt unter den Folgen der Moderne ausgewiesen haben. Daten bieten, anders gesagt, eine Reduktion der Komplexität; denn sie stehen nicht nur für die Verfügbarkeit der für das weitere Handeln notwendigen Informationen. Sie bringen zugleich eine Hierarchisierung der verfügbaren Informationen in Gang und implizieren also Relevanzsetzungen auf der Folie einer algorithmischen Rationalität. So wird der Algorithmus, der die Daten des eingangs angeführten Konzentrationstrackers auswertet, von vornherein bestimmte Daten berücksichtigen und andere nicht, um damit das gewünschte unterrichtliche Phänomen der Aufmerksamkeit zu isolieren. Mit den drei Aspekten der Objektivierung, Hierarchisierung und Kontingenzreduktion können wir uns vor Augen führen, wie die Daten in die Vorstellung eines erfüllten und vollendeten Selbst hineinspielen, deren Legitimierungskraft sich steigert.

Gemeint ist, dass die Daten ein symbolisch-imaginäres Amalgam des Selbst erzeugen, das wir bereits im Eingangsteil unseres Beitrags mit dem Stichwort der «digitalen Aura» aufgenommen haben (Selke, 2016a). Die Beobachtung über die erhobenen Daten wird als Hoffnung der Ermöglichung eines besseren Selbst aufgeladen, so dass das Selbst sich mit dieser digitalen Konstruktion identifiziert. Diese Subjektivierung (siehe den Beitrag von Schröder in diesem Heft) ist der entscheidende Mechanismus, der die Optimierung des Selbst überhaupt zu einer Selbstoptimierung werden lässt. Es geht um den Moment der eigenen Zurechnung zum Selbst.

Das enge und dennoch komplexe Verhältnis zwischen Selbst und dem, was unter «digitaler Aura» oder auch, mit Floridi gesprochen, der «Infosphere» (Floridi 2016) verstanden werden kann, verweist nicht zuletzt auf ein Spannungsfeld von Ungewissheit und Sicherheit, wobei Momente der Ökonomisierung und der Überwachung das Verhältnis mitbestimmen. Entscheidend ist, dass in die Datenvermittlungen des Selbst immer schon Akteurinnen und Akteure mit ökonomischen und politischen Interessen eingebunden sind, die für das Selbst intransparent sind (Turow 2011). Mit Blick auf Sicherheit verweisen wir auf das, was Selke als Sousveillance definiert (Selke 2015) bzw. auf die im Eingangsbeispiel behandelte digital gestützte Überwachung von Schülerinnen und Schülern durch ihre Lehrkräfte und Eltern (Lupton und Williamson 2017) im Sinne einer «intimate surveillance». 
Sicherlich betreffen diese Phänomene nicht nur die Subjekte von Lernen und Bildung, sondern auch die pädagogisch Handelnden. In Bezug auf Elternschaft haben z. B. Blum-Ross und Livingstone (2017) am Beispiel des «Sharenting» (d. h. Eltern, die über das Elternsein bloggen) die Grenzen des digitalen Selbst und seine Komplexität verdeutlicht. Das «self-as-parent» konstruiert sich im Spannungsfeld zwischen individuellen und relationalen Aspekten zwischen Eltern, Kind, Familie und Gesellschaft. Die Reichweite und Zugehörigkeit der Daten (zum Kind oder zu den Eltern im Elternsein) bleiben dabei eigentümlich opak; denn diese Daten sind einerseits Teil einer weitreichenden Vernetzung und andererseits hängen die Daten an den «autonomen» und individuellen Useraccounts, nach denen Plattformen reguliert sind (Blum-Ross und Livingstone 2017). Um Individuen zu überwachen oder unter Bedingungen ökonomischer Interessen einzuordnen und zu bewerten, ist die Annahme eines individuellen Selbst eine Voraussetzung, die in digitalen Strukturen automatisch angelegt ist. Im gleichen Moment kann diese individuelle Ausrichtung auch nur als nötige Organisationsstruktur verstanden werden, um im Folgenden sozial zu sortieren und einzuordnen (Lyon 2003; Mann und Matzner 2019). Hier nun vollzieht sich im Zuge von Big Data eine Verallgemeinerung bis ins kleinste Detail, welche die vorgebliche Individualität als Trugbild entlarvt.

Während in den modernen erziehungswissenschaftlichen und philosophischen Verständigungen des Selbst eine, wie wir oben ausführten, theoretische Unschärferelation zu finden ist, geben datengestützte Darstellungen des Selbst vor, individuell auf die Einzelne oder den Einzelnen abgestimmt zu sein und erzeugen so eine Sicherheit ihrer eigenen Zuschreibungen, auch wenn diese mitunter als fabriziert erscheinen (z. B. bei bearbeiteten Fotos). Ungeachtet der selbstvergewissernden Objektivierungen gehen aus der Datafizierung neue Ungewissheiten hervor. Jedes so sicher erscheinende Datum kann in seiner Bedeutung für die eigene Identität wieder zu einer Quelle der Unsicherheit werden; denn Daten bedürfen der Interpretation. Ausserdem bilden sie Momente in einem zeitlichen Fluss ab. So kann gerade die Aufgabe, die eigene Leistungsfähigkeit zu optimieren, zu einer Angelegenheit des Rätsels bzw. des Selbstzweifels werden. Wie soll bspw. dem Nachlassen der Leistungsfähigkeit begegnet werden, nachdem zuvor doch alles so gut lief?

Die Erklärungskraft der Daten und die Ungewissheit, wie sie für einen selbst zu nehmen sind, stehen im Unverhältnis. ${ }^{2}$ Sie bringen eine Lücke zum Vorschein, welche die Positivität der Daten zu einer Vorspiegelung werden lässt, in der das Selbst vor seinem möglichen Verlust steht. Die Positivität der Daten bei gleichzeitiger Unmöglichkeit, in diesen Daten vollständig aufzugehen, bedingt eine Heimsuchung, in der das Selbst sich erneut vor die Aufgabe einer Bewährung gestellt sieht. Dabei geben

2 Dies steht zunächst in keinem Widerspruch dazu, dass sich sukzessive Normalisierungen, z. B. im Hinblick auf Leistungsfähigkeit, Gesundheit und Gefühle vollziehen (vgl. Vormbusch 2016). Letztlich wird sich auch über den Einsatz der Stirnbänder an Schulen in China ein Normkorridor einer zu erwartenden Leistungsfähigkeit herauskristallisieren. 
weder die Daten noch die an sie anschliessenden Empfehlungen Auskunft darüber, ob man sich auf dem Pfad seines Optimums bewegt. Das Gegenteil ist eher der Fall: Das Selbst steht im Risiko der Abweichung oder der Abirrung vom Optimum des Selbst.

Problematisch an dieser Situation ist - hier folgen wir Selke (2015) -, dass es vor allen Dingen die Anbietenden der Vermessungstechnologien sind, die das Selbst in ein Feld sich erneuernder Optimierungsszenarien führen. Dies verdeutlicht erneut die ökonomische Perspektive, die allgemein bezüglich Praxen von Selbstoptimierung (siehe Selke 2016a, 2016b) und für formale Bildungskontexte im Besonderen (siehe Williamson 2017) wirksam ist und die einer Problematisierung bedarf. Die Effizienzsteigerung folgt ökonomischen Logiken und konstituiert den «Körper als Baustelle», als defizitär und bearbeitungsbedürftig. Dies führt den ökonomischen Logiken folgend jedoch dazu, dass die körperliche Baustelle nie fertig gestellt werden soll, um eine fortwährende Effizienzsteigerung in Aussicht zu stellen. Das Versprechen der Optimierung ist an dieser Stelle trotz der Suggestion der Daten, die Welt verfügbar zu machen, ebenso flüchtig, wie Bauman es der Moderne an sich zuschreibt. Das deterministische Ziel der Technologien bleibt mystifiziert und phantasmatisch (Campolo und Crawford 2020).

In dem, was Campolo und Crawford (2020) als «enchanted determinism» beschreiben, zeichnet sich eine Herausforderung der datengestützten Selbstoptimierung ab. Die Technologien manifestieren sich aus einer «combination of predictive accuracy and mysterious or unexplainable properties», die darin mündet, dass innen «transcendent, superhuman capacities» (ebd., 1) zugeschrieben werden. Gleichzeitig beanspruchen sie "power without responsibility». Die Verantwortung für den Erfolg der Effizienzsteigerung verweilt trotz aller Versprechen und Versuchungen der Verantwortungsabgabe beim Individuum selbst (Bauman und Obirek 2015).

Den Anspruch theoretischer Unschärferelationen in der Bestimmung des Selbst in Rechnung zu stellen, lässt sich somit nicht nur als Aufgabe für Theorien des Selbst formulieren, sondern ist auf die Theorien datengestützter Selbstoptimierung auszudehnen. In der Zusammenführung der vorausgehenden modernisierungstheoretischen Skizze und den Praxen datengestützter Selbstoptimierung lassen sich sowohl Momente des Entzogenen, des Flüchtigen, des Ungewissen als auch der Sicherheit, Rationalisierung und Objektivierung diskutieren. Die Metaphern des Phantomhaften und des Auratischen bilden einen Anhaltspunkt, um die Widersprüche und paradoxalen Logiken der datengestützten Selbstoptimierung herauszuarbeiten. Im Folgenden wollen wir den modernisierungstheoretisch gelagerten Blick auf die datengestützte Selbstoptimierung auf drei Ebenen bündeln: Erstens in Reflexion auf die Suche nach dem Selbst, welche die aktuellen Bedingungen der Datafizierung vorantreibt. Wir plädieren hier dafür, kultur- und sozialwissenschaftliche Beiträge zum digitalisierten Selbst für die erziehungswissenschaftliche Reflexion zu erschliessen. Zweitens 
sehen wir die Notwendigkeit, dem Thema der Datafizierung in seinen unterschiedlichen Hinsichten für pädagogische Prozesse und Praxen nachzugehen. Im Rahmen eines dritten Gesichtspunkts gehen wir auf den Möglichkeitssinn von Theorie bzw. von Bildungstheorie ein. Dieser Möglichkeitssinn, der auf ein Anders-denken-Können zielt, kann im Kontext datengestützter Selbstoptimierung kaum überschätzt werden.

\section{Zur Konstitution und Kritik pädagogischen bzw. erziehungswissenschaftlichen Wissens}

Ein wichtiges Ergebnis unseres Beitrags ist, dass die Suche nach dem Selbst als Motor der datengestützten Selbstoptimierung gesehen werden kann, anstatt diese auszubremsen (Vormbusch 2016). «Das Projekt, zu dem sich das Subjekt befreit, erweist sich heute selbst als Zwangsfigur. Es entfaltet Zwänge in Form von Leistung, Selbstoptimierung und Selbstausbeutung» (Han 2013, 65-66). In diesem Sinn treibt die Suche nach dem Selbst nicht nur die eigene Optimierung voran, sondern auch die Datafizierung gesellschaftlicher Bedingungen an sich. Das Selbst erscheint nie als vollendet; es muss sich immer weiter verändern bzw. optimieren, wie im Bezug zu Bauman und Selke gezeigt wurde. Technologien der Selbstvergewisserung versprechen, diese Optimierung zu unterstützen: in ökonomischer Hinsicht, indem quantifizierbare Aspekte des Selbst objektiviert, vermessen und vergleichbar gemacht werden können, und in kultureller Hinsicht, indem Möglichkeitsräume zur Selbstentfaltung und Selbstentdeckung suggeriert werden (Vormbusch 2016). Aus dieser Bestandsaufnahme ziehen wir die Schlussfolgerung für die Erziehungswissenschaft, dass sie weitgehend kultur- und sozialwissenschaftliche Analysen von Optimierung und Datafizierung aufnehmen und rezipieren muss. Wir kritisieren damit, dass die Diskussion um digitale Technologien auf eng gefasste Vorstellungen von Funktionalität, Praktikabilität und Effizienzsteigerung pädagogischer Interventionen begrenzt bleibt. Demgegenüber scheint es angezeigt, analytisch zu bestimmen, was unter den Bedingungen einer datengestützten Selbstoptimierung als Selbst zu verstehen ist.

Selke (2016b) hat angemerkt, dass die digitale Aura den Menschen auch über das Leben hinweg zu überdauern vermöge. Was bedeuten solche Modulationen der (Un-) Endlichkeit für die Aufgabe theoretischer Beschreibung? Durch die datengestützte Selbstoptimierung entstehen neue Formen der Individualität, die nicht entlang der bisherigen Vorstellungen des Individuums beschrieben werden können. Logiken und Formen der Vernetzung werden aktuell unter den Konzepten von «Dividuum» sowie «Dividualität» diskutiert (Savat 2013; Ott 2015). Für die von uns vorgeschlagene erste Ebene der Reflexion wird zukünftig eine wichtige Aufgabe darin bestehen, Phänomene der Datafizierung und relational dazu die Formierungen des Selbst (als Individuum, als Lernende etc.) zum Gegenstand weiterer Analysen zu machen. Wir sind mit einer komplexen Veränderungsdynamik pädagogischen und erziehungswissenschaftlichen Wissens konfrontiert. 
Damit gehen wir zu einer zweiten Reflexionsebene über, die sich darauf richtet, dass die Veränderungen nicht nur das Selbst der Lernenden betreffen, sondern das Selbstverständnis aller beteiligten Akteurinnen und Akteure. Der eingangs beschriebene Beitrag des Wallstreet Journal beginnt mit der Aussage: «Teachers at this primary school in China know exactly when someone isn't paying attention» (WSJ 2019, 0:05-0:11). Dieser Satz impliziert eine veränderte Haltung zum Unterrichten und also zur Konstitution des unterrichtlichen Lehr-Lern-Raums. Schülerinnen und Schüler als auch Lehrerinnen und Lehrer verändern ihr Verhalten in Anlehnung daran, dass Aufmerksamkeit als messbar dargestellt wird. Das Beispiel verdeutlicht, dass im Angesicht gesellschaftlicher Transformationen auch pädagogisches Wissen transformiert wird. Die Auseinandersetzung aus einer genuin pädagogischen Perspektive heraus ist dabei unabdingbar, da sie sowohl im Beitrag zur Theoriebildung als auch in Bezug zur pädagogischen Praxis Fragen von Ungleichheiten, Orientierungsoptionen und Selbst- und Weltverhältnissen im Kontext der vorhandenen Strukturen offenlegen und diskutierbar machen kann (Hebert 2017).

Besonders markant ist u. E., wie Lehrkräfte oder andere pädagogische Fachkräfte - unterstützt durch Datensets - eine veränderte Vorstellung von dem erhalten, was sie eigentlich über ihre Schülerinnen und Schüler wissen und wissen (könn[t]en). Gegenwärtig ist die erziehungswissenschaftliche Diskussion sehr stark durch eine Orientierung an Medienkompetenz geprägt; es stehen also instrumentell-qualifikatorische Aspekte im Vordergrund. Angehende Lehrkräfte sollen bspw. den Umgang mit digitalen Tools für eine optimale pädagogische Diagnostik lernen. Sie müssen aber auch durchdringen, wie sich die Vorstellung von einer Diagnostik durch den Einsatz dieser Instrumente verschiebt. Es gilt kritisch-theoretisch zu diskutieren, wie durch digitale Transformationsprozesse die Möglichkeiten des pädagogischen Handelns verändert werden (Niesyto 2017). Wie konstituiert sich ein pädagogisch professionelles Handeln, wenn der Handlungsanlass ein Datenset ist? Wie richtet sich die Vorstellung der eigenen pädagogischen Verantwortung neu aus auf der Grundlage von Daten und ihrer algorithmischen Auswertung? (vgl. Fenwick und Edwards 2016)

Mit der Argumentation, dass theoretisches Erschliessen in Zusammenhang mit der Ambivalenz aus Sicherheit und Unsicherheit im Zuge von Datafizierung als wichtiger Teil erziehungswissenschaftlicher Auseinandersetzung zu verstehen ist, kommt eine dritte Reflexionsebene in den Blick. Angelehnt an Horkheimers Kritik des traditionellen Verständnisses von Wissenschaft besteht die Aufgabe von Theorie nicht nur darin, Gegenstände abzubilden und zu beschreiben. Vielmehr bietet Theorie die Möglichkeit, pädagogische Verhältnisse offenzulegen und sie darüber in andere Denkkontexte einzustellen - sie also als anders möglich zu konturieren. Der Einsatz einer Bildungstheorie nach der «humanistischen Illusion» (Schäfer 1996) besteht dementsprechend gerade im Versuch, der Verunsicherung im Verhältnis zum Gegenstand der theoretischen Bestimmung gerecht zu werden. 
Da die erziehungswissenschaftliche Theoretisierung des Selbst dafür relevant ist, wie Menschen sich und ihre Veränderungsmöglichkeiten bestimmen, ist das, was wir als theoretische Unschärferelation in der Bestimmung des Selbst bezeichnet haben, entscheidend dafür, den Raum des Anders-Möglichen zu eröffnen. Bildungstheorie stellt dementsprechend einen kritischen und de-identifizierenden Theorietypus dar, der auf die Engführungen datengestützter Selbstoptimierungstechnologien hinweist. Wenn sich die im Eingangsbeispiel genannten Lehrkräfte in China klar machen, dass es die Konzentrationstracker auch auf eine datengestützte Selbstoptimierung ihrer selbst als Lehrkräfte anlegen, könnte daraus die bildungstheoretisch gehaltvolle Frage hervorgehen, wer wir im Lernen von anderen sein können. Diese Frage ist für das pädagogische Selbstverständnis konstitutiv und sie könnte den Ausgangspunkt für eine nötige breitere gesellschaftliche Auseinandersetzung sein, gerade auch da, wo immer mehr Daten ins Spiel kommen.

\section{Literatur}

Bauman, Zygmunt. 2012. Liquid Modernity. Cambridge: Polity Press.

Bauman, Zygmunt. 2019. Retrotopia. Cambridge: Polity Press.

Bauman, Zygmunt, und Benedetto Vecchi. 2004. Identity. Cambridge: Polity Press.

Bauman, Zygmunt, und Stanislav Obirek. 2015. On the World and Ourselves. Cambridge: Polity Press.

Behrens, John T., und Kirsten DiCerbo. 2014. «Harnessing the Currents of the Digital Ocean». In Learning Analytics: From Research to Practice, herausgegeben von Larusson, J. A., und B. White, B., 39-60. Wiesbaden: Springer. https://doi.org/10.1007/978-1-4614-3305-7_3.

Blum-Ross, Alicia, und Sonia Livingstone. 2017. «Sharenting: parent blogging and the boundaries of the digital self». Popular Communication 15 (2): 110-125. http://doi.org/10.1080/1 5405702.2016.1223300.

Breidenstein, Georg, und Christiane Thompson. 2014. «Schulische Leistungsbewertung als Praxis der Subjektivierung». In Interferenzen. Perspektiven kulturwissenschaftlicher Bildungsforschung, herausgegeben von Christiane Thompson, Kerstin Jergus, und Georg Breidenstein, 89-109. Weilerswist: Velbrück.

Butler, Judith. 2001. Psyche der Macht. Das Subjekt der Unterwerfung. Frankfurt a. M.: Suhrkamp.

Campolo, Alexander, und Kate Crawford. 2020. «Enchanted Determinism: Power without Resonsiblity in Artifical Intelligence». Engaging Science, Technology and Society 6: 1-19. https://doi.org/10.17351/ests2020.277.

Fenwick, Tara, und Richard Edwards. 2016. «Die Auswirkungen digitaler Technologien auf professionelle Verantwortung und Ausbildung». Berliner Debatte Initial 27 (1): 6-21.

Floridi, Luciano. 2016. The Fourth Revolution: How the Infosphere is Reshaping Human Reality. Oxford: Oxford University Press. 
Foucault, Michel. 1977. Der Wille zum Wissen. Frankfurt a. M.: Suhrkamp.

Han, Byung-Chul. 2013. Im Schwarm - Ansichten des Digitalen. Berlin: Matthes \& Seitz.

Hebert, Estella. 2017. «Machtstrukturen im Kontext von Überwachung im Internet und deren Relevanz für die Pädagogik». In Das umkämpfte Netz, herausgegeben von Ralf Biermann, und Dan Verständig, 67-79. Heidelberg: Springer VS Verlag. https://doi.org/10.1007/9783-658-15001-2_5.

Hehl, Walter. 2008. Trends in der Informationstechnologie. Von der Nanotechnologie zu virtuellen Welten. Zürich: vdf Hochschulverlag.

Kitchin, Rob. 2014. «Big Data, New Epistemologies and Paradigm Shifts». Big Data and Society 1 (1): 1-12. https://doi.org/10.1177/2053951714528481.

Lupton, Deborah, und Ben Williamson. 2017. «The datafied child: the dataveillance of children and implications of their rights». New Media and Society 19 (5): 780-794. https://doi. org/10.1177/1461444816686328.

Lyon, David. 2003. Surveillance as Social Sorting - Privacy, Risk and Digital Discrimination. London: Routledge.

Mann, Monique, und Tobias Matzner. 2019. «Challenging algorithmic profiling: The limits of data protection and anti-discrimination in responding to emergent discrimination». Big Data \& Society: 1-11. https://doi.org/10.1177/2053951719895805.

Mau, Steffen. 2018. Das metrische Wir: über die Quantifizierung des Sozialen. Berlin: Suhrkamp.

Meyer-Drawe, Käte. 1990. Illusionen von Autonomie. München: Kirchheim.

Moorstedt, Tobias. 2013. «Erscanne dich selbst». In Big Data. Das neue Versprechen der Allwissenheit, herausgegeben von Heinrich Geiselberger, 67-75. Berlin: Suhrkamp.

Niesyto, Horst. 2017. «Medienpädagogik und digitaler Kapitalismus. Für die Stärkung einer gesellschafts- und medienkritischen Perspektive». MedienPädagogik: Zeitschrift für Theorie Und Praxis Der Medienbildung 27 (Spannungsfelder \& blinde Flecken): 1-29. https://doi. org/10.21240/mpaed/27/2017.01.13.X.

Ott, Michaela. 2015. Dividuationen. Theorien der Teilhabe. Berlin: b_books.

Ricken, Norbert. 2011. «An den Grenzen des Selbst». In Inszenierung und Optimierung des Selbst. Zur Analyse gegenwärtiger Selbsttechnologien, herausgegeben von Ralf Mayer, Christiane Thompson, und Michael Wimmer, 239-257. Wiesbaden: VS Verlag. https://doi. org/10.1007/978-3-658-00465-1_12.

Rose, Nikolas. 1999. Powers of Freedom. Cambridge: University Press.

Savat, David. 2013. Uncoding the Digital. London: Palgrave Macmillan.

Schäfer, Alfred. 1996. Das Bildungsproblem nach der humanistischen Illusion. Weinheim: Deutscher Studienverlag.

Schäfer, Alfred, und Christiane Thompson, Hrsg. 2018. Angst. Paderborn: Schöningh.

Selke, Stefan. 2009. «Die Spur zum Menschen wird blasser. Individuum und Gesellschaft im Zeitalter der Postmedien». In Postmediale Wirklichkeiten. Wie Zukunftsmedien die Gesellschaft verändern, herausgegeben von Stefan Selke, und Ulrich Dittler, 13-57. Hannover: Heise. 
Selke, Stefan. 2015. «Lifelogging und die neue Taxonomie des Sozialen». In Big Data und Medienbildung. Zwischen Kontrollverlust, Selbstverteidigung und Souveränität in der digitalen Welt, herausgegeben von Harald Gapski, 95-110, Schriftenreihe zur digitalen Gesellschaft NRW, Band 3. Düsseldorf/München: kopaed.

Selke, Stefan. 2016a. «Einleitung. Lifelogging zwischen disruptiver Technologie und kulturellem Wandel». In Digitale Selbstvermessung und Lebensprotokollierung zwischen disruptiver Technologie und kulturellem Wandel, herausgegeben von Stefan Selke, 1-21. Wiesbaden: Springer VS. https://doi.org/10.1007/978-3-658-10416-0_1.

Selke, Stefan. 2016b. «Vom vermessenen zum verbesserten Menschen? Lifelogging zwischen Selbstkontrolle und Selbstoptimierung». In Körperphantasien. Technisierung - Optimierung - Transhumanismus, herausgegeben von Andreas Beinsteiner, und Tanja Kohn, 131151. Innsbruck: Innsbruck University Press 2016 (Medien - Wissen - Bildung). https://doi. $\operatorname{org} / 10.25969 /$ mediarep/1200.

Tester, Keith. 2002. «Paths in Zygmunt Bauman's Social Thought». Thesis Eleven 70: 55-71. https://doi.org/10.1177/0725513602070001006.

Thompson, Christiane, Jörg Zirfas, Wolfgang Meseth, und Thorsten Fuchs, Hrsg. 2021. Erziehungswirklichkeiten in Zeiten von Angst und Verunsicherung. Weinheim: Beltz.

Turow, Joseph. 2011. The Daily You. New Haven/London: Yale University Press.

Vormbusch, Uwe. 2016. «Taxonomien des Selbst». In Leben nach Zahlen. Self-Tracking als Optimierungsprojekt?, herausgegeben von Stefanie Duttweiler, Robert Gugutzer, Jan-Hendrik Passoth, und Jörg Stübing, 45-59. Bielefeld: transcript. https://doi. org/10.1515/9783839431368-002.

Wallstreet Journal (WSJ). 2019. "How China is Using Artificial Intelligence in Classrooms». https://www.youtube.com/watch?v=JMLsHI8aV0g.

Williamson, Ben. 2017. «Decoding ClassDojo: psycho-policy, social-emotional learning and persuasive educational technologies». Learning, Media and Technology 42 (4): 440-453. https://doi.org/10.1080/17439884.2017.1278020.

Wimmer, Michael. 2011. «Das Selbst als Phantom». In Inszenierung und Optimierung des Selbst. Zur Analyse gegenwärtiger Selbsttechnologien, herausgegeben von Ralf Mayer, Christiane Thompson, und Michael Wimmer, 295-321. Wiesbaden: VS Verlag. https://doi. org/10.1007/978-3-658-00465-1_14. 